\title{
Surface nanodrops and nanobubbles: a classical density functional theory study
}

\author{
Peter Yatsyshin $^{1} \dagger$ and Serafim Kalliadasis ${ }^{1}$ \\ ${ }^{1}$ Department of Chemical Engineering, Imperial College London, London, SW7 2AZ, UK
}

(Received $\mathrm{xx}$; revised $\mathrm{xx}$; accepted $\mathrm{xx}$ )

\begin{abstract}
We present a fully microscopic study of interfacial thermodynamics of nanodrops and nanobubbles, adsorbed on flat substrates with first-order wetting. We show that both nanodrops and nanobubbles are thermodynamically accessible in regions, demarcated by the spinodals of planar wetting films, with nanobubbles occupying a relatively bigger portion of the phase space. While nanodrops can be described as near-spherical caps of Laplace radius, the radius of nanobubbles is very different from the Laplace value. Additionally, nanobubbles are accompanied by a thin gas film adsorbed on the substrate. By computing the interface binding potential, we relate the sphericity of nanodrops to the thin-thick liquid film coexistence (prewetting transition), whereas nanobubble shapes are determined only by the decay of the fluid-substrate forces.
\end{abstract}

Key words: Nanodroplets, nanobubbles, wetting, binding potential, disjoining pressure

\section{Introduction}

Sessile drops and bubbles with characteristic dimensions on the nanometer scale play a central role in a wide spectrum of applications, from inkjet printing and vapourliquid-solid growth processes to micro-/nanofluidics and the design and operation of labon-a chip devices. But their theoretical understanding remains incomplete and several fundamental aspects still elude us. This is largely due to the challenging physics of such small objects, often revealing sensitive dependence on the nature of intermolecular forces, surface wetting properties (Bonn \& Ross 2001) and interface fluctuations (Rascón \& Parry 2000). In particular, using a model disjoining pressure, Svetovoy et al. (2016) recently showed that surface-interface interactions significantly affect the shapes and contact angles of nanobubbles. Recent reviews by Theodorakis \& Che (2019), Qian et al. (2019) and Lohse \& Zhang (2015) discuss the latest experimental approaches, simulation studies and capillary models of surface nanodrops and nanobubbles. Interesting and promising fully microscopic approaches for computing the disjoining pressure of adsorbed liquid films were recently developed by MacDowell (2011), MacDowell et al. (2014), Nold et al. (2014). Using classical density functional theory (DFT), Hughes et al. (2017) and Yin et al. (2019) proposed a method for computing the binding potential which captures the near-wall behaviour of the fluid density. The latter is obtained from a constrained minimisation of the DFT grand free energy functional, subject to an additional requirement that the density profile must have a given thickness of the liquid film.

In the present paper we use DFT to consistently incorporate the existence of the surface

$\dagger$ Email address for correspondence: p.yatsyshin@imperial.ac.uk 
phase transitions, particularly the pre-wetting transition, and uncover their effects on the formation and stabiliity of nanodrops and nanobubbles. This allows us to identify thermodynamic accessibility regions of nanodrops and nanobubbles adsorbed on flat walls. Using DFT we propose a method for computing the interface binding potential, which is based on computing the work of adsorbate formation from the free energy functional. Full unconstrained minimisation of DFT allows us to capture the correct long-ranged asymptotes of the density profiles, which are known to affect the binding potential. It also allows us to treat liquid and gas adsorption consistently within the same framework and uncover a very intriguing relationship between film adsorption and drop/bubble formation.

The intermolecular fluid-fluid and fluid-substrate interactions are considered to be of Lennard-Jones (LJ) type, with potential well depth $\varepsilon$ and range $\sigma$ :

$$
\varphi_{\varepsilon, \sigma}^{6-12}(r)=4 \varepsilon\left[-\left(\frac{\sigma}{r}\right)^{6}+\left(\frac{\sigma}{r}\right)^{12}\right] .
$$

Such long-range forces typically lead to first-order wetting transitions and planar liquid film coexistence. In the present work we uncover the relations between the intriguing equilibrium properties of nano-drops and -bubbles and the physics and stability of planar adsorbed wetting films. We stay within the mean-field description of wetting, which remains valid for most practical purposes. The role of interface fluctuations and system dimensionality is discussed, e.g., in excellent reviews by Dietrich (1988) and Saam (2009).

In section 2 we employ a non-local microscopic approach based on DFT. It naturally accounts for the bulk liquid-gas coexistence, planar wetting and Young contact angle of macroscopic three-phase contact lines, thus fully capturing the physics of nanodrops and nanobubbles. We proceed to discussing the intriguing differences in the microscopic shapes of nanodrops and nanobubbles. We find that adsorbed nanodrops have nearspherical caps of Laplace radius. At the same time, nanobubbles found in the region of the phase diagram which is symmetric with respect to the liquid-gas coexistence line have radii very different from the Laplace value. Additionally, nanobubbles are accompanied by a thin film of gas adsorbed on the substrate. This leads us to relate classical DFT with Derjaguin's approach to wetting in section 3, where we propose a systematic and transferable method for computing the interface binding potential (antiderivative of disjoining pressure) using DFT adsorption isotherms. The binding potential gives us access to the energy landscape of the contact line and reveals that the near-sphericity of nanodrops is caused by the remnant of thin-thick liquid film coexistence, the socalled planar prewetting transition. At the same time, the flatter shape of nanobubbles is determined by the absence of such film coexistence during gas adsorption. We summarise our findings in section 4 and provide an outlook of potential broader applications of the fully microscopic route to computing the disjoining pressure.

\section{Classical DFT applied to wetting}

At any given temperature $T$ and chemical potential $\mu$ the equilibrium fluid density $\rho(\mathbf{r})$ minimizes a grand free energy functional $\Omega[\rho ; T, \mu]$, which includes terms describing short-range hard-sphere-like molecular repulsions $F_{\mathrm{hs}}[\rho ; T]$, long-range attractions $F_{\text {attr }}[\rho]$ and the contribution from an external potential $V_{\text {ext }}(\mathbf{r})$ :

$$
\Omega[\rho(\mathbf{r}) ; T, \mu]=F_{\mathrm{hs}}[\rho ; T]+F_{\mathrm{attr}}[\rho]+\int V_{\mathrm{ext}}(\mathbf{r}) \rho(\mathbf{r}) \mathrm{d} \mathbf{r}-\mu \int \rho(\mathbf{r}) \mathrm{d} \mathbf{r},
$$


where the integration is carried out over the volume $V$ of the fluid. At equilibrium, equation (2.1) gives the thermodynamic grand potential $\Omega(T, \mu)$. For a uniform fluid, $\Omega(T, \mu)=-P V$, where $P$ is pressure. Over the years, sophisticated approximations have been developed for each respective contribution in equation (2.1), see, e.g., the reviews by Evans (1990), Wu \& Li (2007) and Lutsko (2010). In the present work we use a simple free energy functional which, despite being minimalistic, still captures the essential physics of the liquid-gas interface near attractive substrates in the LJ model of attractions (Sullivan \& Telo da Gama 1986). The intermolecular repulsions are treated within the so-called local density approximation (Pereira \& Kalliadasis 2012):

$$
F_{\mathrm{hs}}[\rho(\mathbf{r})]=\int k_{\mathrm{B}} T \rho(\mathbf{r})\left(\ln \left(\lambda^{3} \rho(\mathbf{r})\right)-1\right) \mathrm{d} \mathbf{r}+\int \psi(\rho(\mathbf{r})) \rho(\mathbf{r}) \mathrm{d} \mathbf{r},
$$

where $k_{\mathrm{B}}$ is the Boltzmann constant, $\lambda$ is the de Broglie thermal wavelength; and $\psi(\rho)$ is the configurational part of the Carnahan-Starling hard sphere fluid free energy (Lutsko 2010):

$$
\psi(\rho)=k_{\mathrm{B}} T \frac{\eta(4-3 \eta)}{(1-\eta)^{2}}, \quad \eta=\pi \sigma^{3} \rho / 6
$$

where $\sigma$ is the hard sphere diameter, which here we consider to be fixed and equal to the range of the LJ potential in equation (1.1). This treatment neglects the weak temperature-dependence of the hard-sphere diameter in thermodynamic perturbation theories (Lutsko 2010). The model of $F_{\text {attr }}[\rho]$ is the most important DFT ingredient in the present analysis. We use the random phase approximation for the direct correlation function of the uniform LJ fluid:

$$
F_{\text {attr }}[\rho(\mathbf{r})]=\frac{1}{2} \int \mathrm{d} \mathbf{r} \int \rho(\mathbf{r}) \rho\left(\mathbf{r}^{\prime}\right) \varphi_{\text {attr }}\left(\left|\mathbf{r}-\mathbf{r}^{\prime}\right|\right) \mathrm{d} \mathbf{r}^{\prime},
$$

where $\varphi_{\text {attr }}(r)$ is a mean-field potential:

$$
\varphi_{\text {attr }}(r)= \begin{cases}0, & r \leqslant \sigma \\ \varphi_{\varepsilon, \sigma}^{6-12}(r), & r>\sigma .\end{cases}
$$

We are interested in planar adsorption, where the LJ substrate occupies a half-space:

$$
V_{\text {ext }}(x)=4 \pi \rho_{0} \varepsilon_{0} \sigma_{0}^{3}\left(-\frac{1}{6}\left(\frac{\sigma_{0}}{H_{0}+x}\right)^{3}+\frac{1}{45}\left(\frac{\sigma_{0}}{H_{0}+x}\right)^{9}\right),
$$

where $\rho_{0}$ is the effective substrate density, and $\varepsilon_{0}$ and $\sigma_{0}$ are the substrate-specific LJ parameters. We have introduced a low- $x$ cutoff parameter $H_{0}$ in equation (2.6) to eliminate the non-physical divergence of $V(x)$ at fluid-substrate contact. This is a purely mathematical device and does not affect the physics of adsorption as was shown in our earlier work (Yatsyshin \& Kalliadasis 2016).

In the present work we investigate the liquid-gas interface and its behaviour near an attractive substrate wall. It is very well known that the physics of the liquid-gas interface is determined by the attractive part of the intermolecular potential (Sullivan \& Telo da Gama 1986). This is captured by the mean-field term in equation (2.4). Although the present DFT model describes the intermolecular attractions in a nonlocal mean-field fashion, which allows us to consider droplets and bubbles as narrow as several tens of molecular diameters, the intermolecular repulsions are treated by a purely local approximation, as can be seen in the repulsive term in equation (2.2). This term neglects the excluded volume correlations. As a result, the density profiles we compute do not exhibit the characteristic near-wall oscillations and layering, which can be captured 
with more refined non-local approximations, such as weighted density or fundamental measure theories (FMT) (Roth 2010), replacing equation (2.2). However, our DFT given by equations (2.2) and (2.4) provides a minimalistic valid approximation, which captures all the important physics of the liquid-gas interface and its interplay with the wall in the range of temperatures above the bulk triple point, which we study here. Moreover, we do not expect the molecular packing effects to be important for the qualitative aspects of wetting by liquid above the fluid bulk triple point, for the simple reason that layering transitions do not interfere with pre-wetting. Similarly, the mean-field nature of our model energy functional implies that our results do not capture effects associated with capillary wave fluctuations. With these shortcomings, our model functional (2.1) - (2.5) provides a suitable microscopic starting point to study the formation of microscopic drops and bubbles. Further discussion of the physical approximations underlying (2.1) $-(2.5)$ and possible approaches to their numerical solution can be found elsewhere, e.g., in Yatsyshin et al. $(2015,2016,2017)$.

The equilibrium density profile $\rho(\mathbf{r})$ satisfies the Euler-Lagrange (EL) equation:

$$
\begin{array}{r}
k_{\mathrm{B}} T \ln \rho(\mathbf{r})+\psi(\rho(\mathbf{r}))+\rho(\mathbf{r}) \psi_{\rho}^{\prime}(\rho(\mathbf{r})) \\
+\int \rho\left(\mathbf{r}^{\prime}\right) \varphi_{\text {attr }}\left(\left|\mathbf{r}-\mathbf{r}^{\prime}\right|\right) \mathrm{d} \mathbf{r}^{\prime}+V_{\text {ext }}(\mathbf{r})-\mu=0,
\end{array}
$$

where $\psi_{\rho}^{\prime}(\rho)$ is the derivative of $(2.3)$ with respect to $\rho$. In the bulk, $V_{\text {ext }}(\mathbf{r}) \rightarrow 0$ and $\rho(\mathbf{r}) \rightarrow \rho_{\mathrm{b}}$ (bulk density), and equations (2.1) and (2.7) give us the pressure and chemical potential:

$$
\begin{array}{r}
P\left(T, \rho_{\mathrm{b}}\right)=\rho_{\mathrm{b}} k_{\mathrm{B}} T \frac{1+\eta+\eta^{2}-\eta^{3}}{(1-\eta)^{3}}-\frac{16 \pi}{9} \rho_{\mathrm{b}}^{2} \sigma^{3} \varepsilon, \\
\mu\left(T, \rho_{\mathrm{b}}\right)=k_{\mathrm{B}} T \ln \rho_{\mathrm{b}}+\psi\left(\rho_{\mathrm{b}}\right)+\rho_{\mathrm{b}} \psi_{\rho}^{\prime}\left(\rho_{\mathrm{b}}\right)-\frac{32 \pi}{9} \rho_{\mathrm{b}} \sigma^{3} \varepsilon .
\end{array}
$$

At bulk liquid-gas coexistence (saturation), $P\left(T, \rho_{\mathrm{l}}\right)=P\left(T, \rho_{\mathrm{g}}\right)=P_{\text {sat }}(T)$ and $\mu\left(T, \rho_{\mathrm{l}}\right)=\mu\left(T, \rho_{\mathrm{g}}\right)=\mu_{\text {sat }}(T)$, where $P_{\text {sat }}$ and $\mu_{\text {sat }}$ are the saturation pressure and chemical potential. The boundaries of phase metastability are given by the spinodals: $\partial P /\left.\partial \rho\right|_{\rho_{\mathrm{g}}, \rho_{1}}=0$; and the critical point at $T_{\mathrm{c}}$ and $\rho_{\mathrm{c}}$ satisfies $\partial P / \partial \rho_{\mathrm{c}}=\partial^{2} P / \partial \rho_{\mathrm{c}}^{2}=0$.

In what follows, we adopt a system of units where the hard-sphere diameter $\sigma$ and the well depth $\varepsilon$ in equation (2.5) are set as units of length and energy, respectively. This leads, e.g., to the bulk critical temperature $T_{\mathrm{c}}=1.006 \varepsilon / k_{\mathrm{B}}$. We also fix the parameters of $V_{\text {ext }}$ as $\varepsilon_{0}=0.4, \sigma_{0}=2$ and $H_{0}=5$, which gives a first-order wetting wall with a welldefined prewetting line and a relatively high wetting temperature $T_{\mathrm{w}} \approx 0.915$. In bulk metastability regions it is convenient to use "disjoining" chemical potential $\Delta \mu(T)=$ $\mu-\mu_{\text {sat }}(T)$. Also, given $T$ and $\mu$, we shall refer to $\Delta \rho(T, \mu)=\rho_{\mathrm{b}}-\tilde{\rho}_{\mathrm{b}}$ as the difference between the densities of phases, stable and metastable in the bulk, respectively. When $\Delta \mu \neq 0$, both $\rho_{\mathrm{b}}$ and $\tilde{\rho}_{\mathrm{b}}$ solve equation (2.9) at the same $T$, but $P\left(T, \rho_{\mathrm{b}}\right) \geqslant P\left(T, \tilde{\rho}_{\mathrm{b}}\right)$.

\subsection{Adsorption of film, drops and bubbles}

Near a planar wall, the fluid density depends on the distance $x$ to the wall. For LJ forces, $\rho(x)-\rho_{\mathrm{b}}=\mathcal{O}\left(x^{-3}\right)$, as $x \rightarrow \infty$. Due to the combination of wall-fluid and solvation forces, the wall can adsorb a layer of thickness $l$ of a new phase. For liquid and gas adsorption, the respective $l=l_{\text {liq }}$ and $l=l_{\text {gas }}$ are given by the same expression, see figure $1(\mathrm{c})$ :

$$
l(T, \mu)=\frac{\int_{0}^{\infty}\left(\rho(x)-\rho_{\mathrm{b}}\right) d x}{\Delta \rho(T, \mu)},
$$


where $\Gamma(T, \mu)=\int_{0}^{\infty}\left(\rho(x)-\rho_{\mathrm{b}}\right) d x$ is adsorption. The grand free energy density $\omega(x)$ can be found for $\rho(x)$, at $T$ and $\mu$ by rearranging equation (2.1) (Rowlinson \& Widom 1982):

$$
\Omega[\rho(x) ; T, \mu]=A \int_{0}^{\infty} \omega(x ; \rho(x), T, \mu) \mathrm{d} x,
$$

where $A$ is the fluid-wall interface area. The interface tension is given by the integral $\gamma(T)=\int_{0}^{\infty} \omega^{\mathrm{ex}}(x) d x$, where the excess-over-bulk grand free energy density $\omega^{\mathrm{ex}}(x)=$ $\omega(x)+P\left(\rho_{\mathrm{b}}, T\right)$, and decays as $\mathcal{O}\left(x^{-3}\right)$ when $x \rightarrow \infty$. We can thus compute the surface tensions of the saturated liquid and gas with the wall, $\gamma_{\mathrm{wl}}$ and $\gamma_{\mathrm{wg}}$, and the surface tension of the free liquid-gas interface $\gamma_{\mathrm{lg}}$. Off of saturation the Laplace radius of a drop or bubble is $R_{\mathrm{L}}=\gamma_{\mathrm{lg}} / \Delta \mu \Delta \rho$ (Hauge 1992; Rowlinson \& Widom 1982). At saturation, the Young contact angle $\Theta_{\mathrm{Y}}(T)=\cos ^{-1}\left(\left(\gamma_{\mathrm{wl}}-\gamma_{\mathrm{wg}}\right) / \gamma_{\mathrm{lg}}\right)$ is non-zero below $T_{\mathrm{w}}$, and vanishes at $T_{\mathrm{w}}$ as $\mathcal{O}\left(\sqrt{T_{\mathrm{w}}-T}\right)$.

Figure 1 represents the mean-field picture of gas and liquid adsorption. The adsorption isotherms $l_{\text {liq }}(\mu)$ and $l_{\text {gas }}(\mu)$ at $T=0.88<T_{\mathrm{w}}$, plotted in figure $1(\mathrm{a})$, represent the bifurcations of two sets of solutions to the EL equation $(2.7),\{\rho(x)\}_{T}^{\text {gas }}$ and $\{\rho(x)\}_{T}^{\text {liq }}$ respectively, with bulk gas and liquid densities. These can be obtained using arc-length continuation, as discussed by Yatsyshin et al. (2015). Coloured branches denote (meta)stable surface phases, meaning that the fluid density profiles computed at respective $\mu$ are local minima of $\Omega[\rho]$. Grey branches are unstable (respective fluid configurations extremise $\Omega[\rho]$, but are not its minima). Both isotherms diverge at saturation as $\mathcal{O}\left(\Delta \mu^{-1 / 3}\right)$, when $\Delta \mu \rightarrow 0$ from below, where bulk gas is stable and bulk liquid is metastable. In the case of gas adsorption, this is a heterogeneous nucleation of gas, stable in the bulk, on the wall. The unstable branch of the isotherm, where the gas films are thick, corresponds to "critical" nucleation clusters, and the turning point (spinodal) signals an upward shift of the bulk liquid spinodal, induced by the wall. Below the isotherm spinodal, supersaturated liquid simply cannot coexist with the wall. The liquid adsorption isotherm, on the other hand, represents partial wetting, where the adsorbed liquid is metastable in the bulk. Below $T_{\mathrm{w}}, \Theta_{\mathrm{Y}}>0$ and thick liquid films are metastable, meaning that their grand potential is higher than that of the thin films at the same $\mu$. Thin-thick film coexistence is a first-order prewetting transition, happening at $T>T_{\mathrm{w}}$ and $\mu=$ $\mu_{\mathrm{pw}}(T)<\mu_{\mathrm{sat}}$. The unstable branch of the liquid film isotherms, therefore, corresponds to "critical" prewetting clusters. The isotherm spinodals bound the metastability of thin (green) and thick (red) adsorbed liquid film. The thin film spinodal is a downward shift of the bulk gas spinodal: metastable gas cannot coexist with the wall above it. Notice also that the lower branch of the gas film isotherm is a few molecular diameters higher than its liquid film counterpart. This is a packing effect associated with solvation force locally repelling the liquid from the wall.

The phase diagram is depicted in figure $1(\mathrm{~b})$. At different $T$, the spinodals of the surface phases [coloured circles in 1(a)] form respectively coloured spinodal lines in 1(b). As we shall see, these bound the regions (hatched), where surface drops and bubbles are nucleated on the wall. The bulk liquid-gas coexistence line $\mu_{\text {sat }}(T)$ (dashed grey) terminates at the critical point $T_{\mathrm{c}}$, and bulk spinodals (solid grey) extend tangentially from it, demarcating the metastability regions of bulk liquid (below $\mu_{\text {sat }}$ ) and gas (above $\left.\mu_{\text {sat }}\right)$. We reflect the fact that the wall curtails bulk metastability by shading with grey the forbidden region below gas film spinodal and above thick liquid film spinodal, where metastability is impossible. The first-order wetting transition at $\left(T_{\mathrm{w}}, \mu_{\mathrm{sat}}\right)$ marks the coexistence between microscopically thin and macroscopically thick liquid wetting films, and serves as the starting point of the prewetting line $\mu_{\mathrm{pw}}(T)$ (black), which approaches 

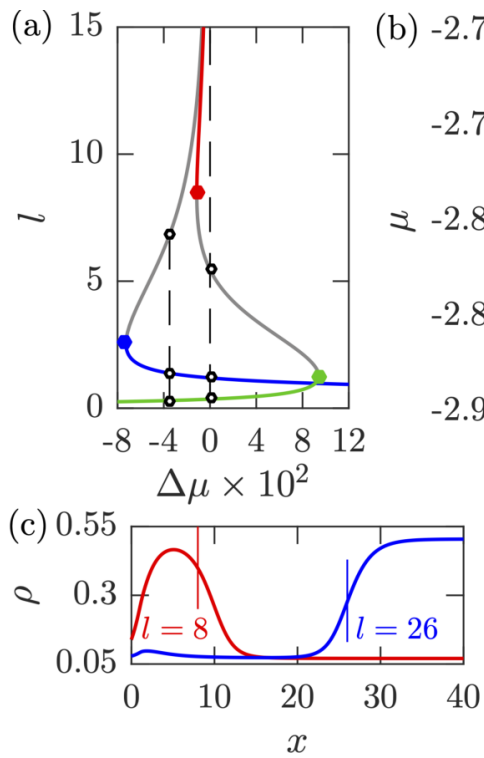
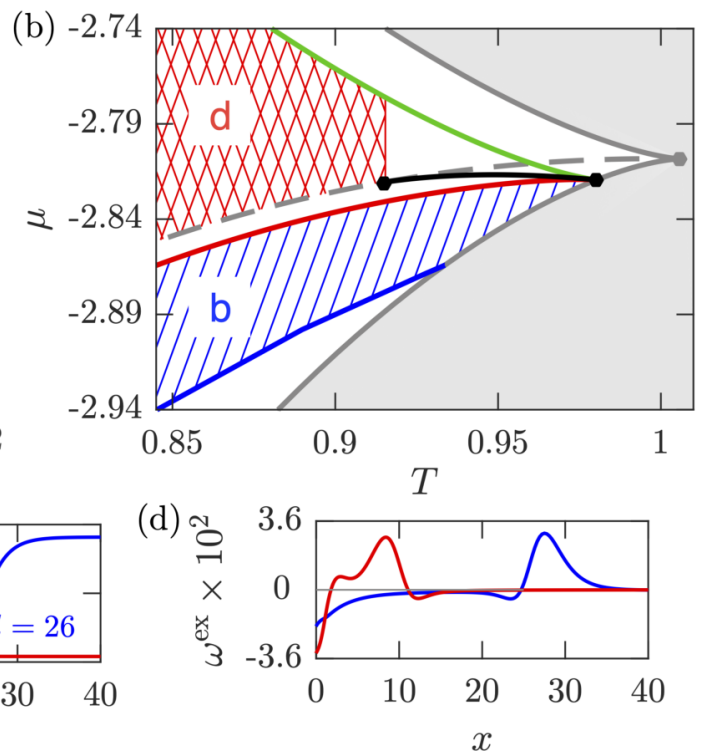

FiguRE 1. (a) Isotherms of liquid and gas adsorption, $l_{\text {liq }}(\mu)$ (green-grey-red curve) and $l_{\text {gas }}(\mu)$ (blue-grey curve), at $T=0.88\left(\Theta_{\mathrm{Y}}=50.6^{\circ}\right)$. Grey parts correspond to unstable adsorbed films. Thin liquid films (green) are stable and thick liquid films (red) are metastable. Thin gas films (blue) are stable. Spinodals are designated by filled circles; their $T$-dependence is shown in (b) by respectively coloured lines. Dashed vertical lines at $\Delta \mu=0$ and $\Delta \mu=-0.035$ are explained in figure 3. (b) Bulk coexistence line $\mu_{\text {sat }}(T)$ (dashed grey) and its spinodals (solid grey), ending at the critical point (grey circle), $T_{\mathrm{c}}=1.006$. Metastability is impossible in the grey-shaded region. Prewetting line $\mu_{\mathrm{pw}}(T)$ (solid black) starts at $T_{\mathrm{w}}=0.915$ and ends at $T_{\mathrm{pw}}^{\mathrm{c}}=0.98$ (both are designated by black circles). Surface drops/bubbles occur in the cross-/single-hatched regions, respectively (also designated by "d" and "b"). (c) and (d) Representative profiles of density [with $l=6$ and $l=26$, marked in (c)] and grand free energy density. Red/blue curves correspond to liquid/gas adsorption.

saturation tangentially as $\mu_{\text {sat }}(T)-\mu_{\mathrm{pw}}(T)=\mathcal{O}\left(\left(T-T_{\mathrm{w}}\right)^{3 / 2}\right)$, when $T \rightarrow T_{\mathrm{w}}$, and terminates at $T_{\mathrm{pw}}^{\mathrm{c}}$, at the prewetting critical point, lying on the bulk gas spinodal. Figures 1 (c) and (d) show sample density profiles of adsorbed liquid (red, $l=8$ ) and gas (blue, $l=26$ ), and the corresponding profiles of excess-over-bulk grand free energy density. Inside adsorbed and bulk phases $\omega^{\mathrm{ex}}(x) \approx 0$, and the fluid-wall and liquid-gas interfaces are each associated with an oscillation of $\omega^{\mathrm{ex}}(x)$ (blue curves). Moreover, DFT captures the complicated interplay between these two interfaces at small $l$ (red curves).

On a planar wall, the DFT EL equation (2.7) can only have film-like solutions. To obtain surface drops/bubbles, we insert a nucleation seed by locally increasing/decreasing the potential well of $V_{\text {ext }}$ by $5 \%$ of its value in $1 \sigma$-vicinity of $x=0$. This perturbation is a purely mathematical device we use to break the symmetry of the EL equation. It does not effect the structure of the adsorbate. A few representative surface drops and bubbles at $T=0.6<T_{\mathrm{w}}$ and $|\Delta \mu| \rightarrow 0$ are shown in figure 2 . Here these configurations are unstable extrema of $\Omega[\rho]$, and thus correspond to critical nucleation clusters of liquid and gas during heterogeneous nucleation on the wall, which acts as the nucleation center. Indeed, we find that the average inner density inside each drop/bubble is $\rho_{\mathrm{b}}(\mu)$.

The shapes of surface drops and bubbles in figure 2 are affected by several factors. The curvature of the liquid-gas interface leads to the $\gamma_{\mathrm{lg}} / R_{\mathrm{L}}$ pressure increment inside the adsorbed phase. Therefore, Laplace radius should provide a good overall estimate of the characteristic dimensions of the drops and bubbles. At the same time, the interface 

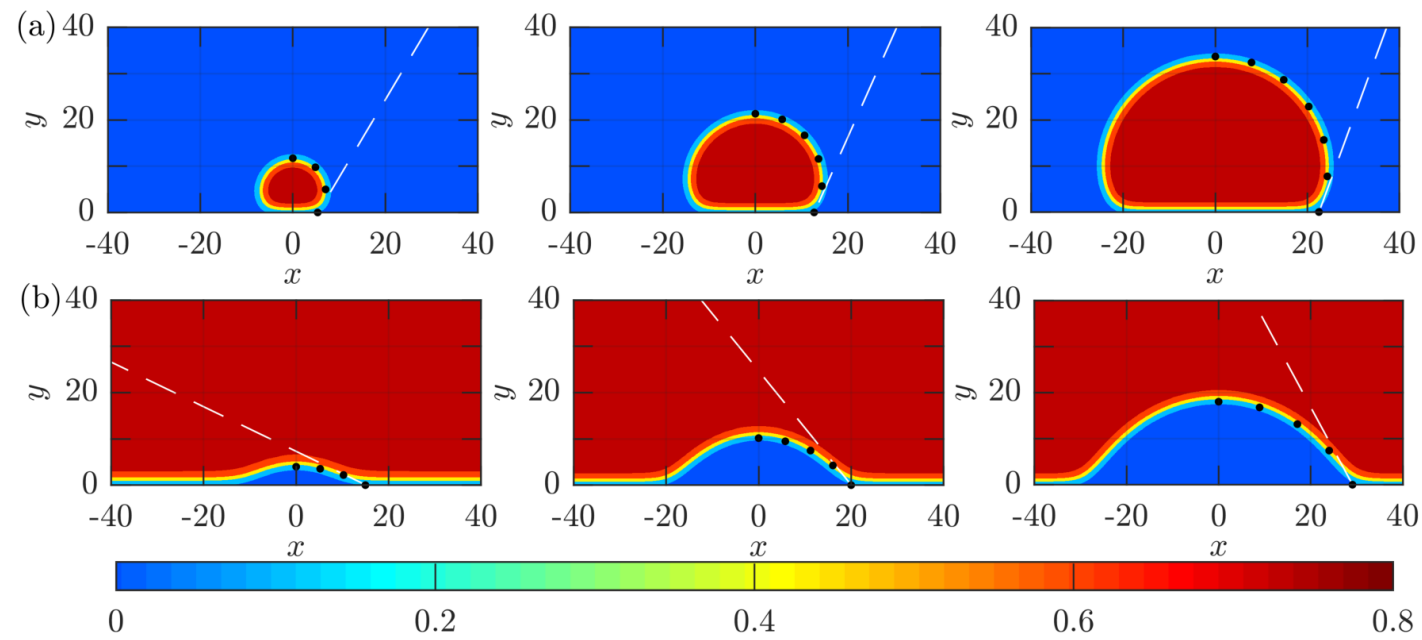

\section{0}

0.2

0.4

0.6

0.8

Figure 2. Density profiles of surface nanodrops (a) and nanobubbles (b) at $T=0.6$ $\left(\Theta_{\mathrm{Y}}=105^{\circ}\right)$, and (left to right) $\Delta \mu= \pm 0.13\left(R_{\mathrm{L}} \approx 8\right), \Delta \mu= \pm 0.07\left(R_{\mathrm{L}} \approx 15\right), \Delta \mu= \pm 0.04$ $\left(R_{\mathrm{L}} \approx 25\right)$, where positive/negative $\Delta \mu$ corresponds to drops/bubbles, respectively. Dotted curves show the arc of a circle, fitted to the liquid-gas interface (density level set at $\left(\rho_{1}+\rho_{\mathrm{g}}\right) / 2$ ) to compute the apparent radii of the drops, $R_{\mathrm{d}}^{*} \approx 8,15,25$ and bubbles, $R_{\mathrm{b}}^{*} \approx 30,25,32$. The apparent contact angles, $\Theta_{\mathrm{d}}^{*}=121^{\circ}, 114^{\circ}, 110^{\circ}$ and $180^{\circ}-\Theta_{\mathrm{b}}^{*}=154^{\circ}, 129^{\circ}, 118^{\circ}$, are computed by drawing a tangent (dashed lines) to the circular fit at a distance $1 \sigma$ from the wall. The values of $\Theta_{\mathrm{d} / \mathrm{b}}^{*}$ of sufficiently small drops/bubbles are highly sensitive to the technical details of the circle fitting procedure. These values are provided here to illustrate the convergence to $\Theta_{Y}$ as the drop/bubble grows.

shape, especially near the wall, is the result of a complicated interplay between the liquidgas and wall-fluid interfaces, such as shown in figure 1(d) for flat films. Indeed, near the substrate, the drop interfaces visibly bend in, whilst the bubble interfaces flatten out, connecting with the thin layer of gas, which covers the outside wall and is induced by the solvation force. This is in agreement with the film adsorption isotherms represented in figure 1(a). Moreover, at small and intermediate sizes, bubbles are significantly flatter than the drops. This happens because the ambient phase interacts very strongly with the substrate in the case of bubbles, "squashing" them.

To test the classical size estimate, given by the Laplace radius, we fit a circle to the liquid-gas interface of surface drops/bubbles and find their "apparent" radii $R_{\mathrm{d} / \mathrm{b}}^{*}$ and contact angles $\Theta_{\mathrm{d} / \mathrm{b}}^{*}$. For figure 2 these are reported in the caption, together with the Laplace radii $R_{\mathrm{L}}$. We introduced a small near-wall cutoff for fitting the circle to avoid the contribution of the "flattening" region near the bubble edges.

Let us note that the values of $R_{\mathrm{L}}$ are very close for drops and bubbles at the same $|\Delta \mu|$. This means that a description in terms of $R_{\mathrm{L}}$, which ignores the microscopic details of fluid-substrate interaction, views drop and bubble adsorption in a completely identical way. Interestingly still, for small drops this description seems to hold up, as we see a remarkable agreement between $R_{\mathrm{d}}^{*}$ and $R_{\mathrm{L}}$ across the drop sizes considered. But the story changes in the case of bubbles where there is hardly any agreement between $R_{\mathrm{L}}$ and the fitted radius of the two smaller bubbles. Clearly, the fluid-substrate interactions neglected by $R_{\mathrm{L}}$ are very important for bubbles. Of course, for larger bubbles $R_{\mathrm{b}}^{*}$ must start catching up with $R_{\mathrm{L}}$ (and we can see the beginning of this trend between the bubbles with $R_{\mathrm{b}}^{*}=25$ and 32 ), because at saturation the Young contact angle must be recovered. Intuitively, it is clear that the denser liquid above the bubble is attracted to the wall, 
squashing the bubble. Indeed, this is the intuition behind the binding potential, which we introduce in the next section. This squashing does not happen for drops, because the gas above the drop is too dilute to squash them. We further note that the approach of apparent contact angles to Young values with growing $R_{\mathrm{d} / \mathrm{b}}^{*}$ is also clear and testifies to the correctly set up DFT computation.

Finally, notice that DFT also elegantly reveals the thermodynamics of drop and bubble nucleation, summarized in figure 1(b). The wall must be dry to act as a nucleation center for liquid. Accordingly, we find surface drops forming at $\left(T<T_{\mathrm{w}}, \mu\right)$ in the red crosshatched region of figure $1(\mathrm{~b})$. The drop radii are minimal on the liquid film spinodal, and diverge at saturation as $\mathcal{O}\left(\left(P\left(T, \rho_{b}\right)-P_{\text {sat }}\right)^{-1}\right)$, according to the Laplace law. Above $T_{\mathrm{w}}$, $\Theta_{\mathrm{Y}}=0$ and the wall impurity would nucleate a planar wetting film, see, e.g. Yatsyshin et al. (2016, 2017). Surface bubbles can be found in a broader blue-hatched region of figure 1(b), above the gas film spinodal, where their radii are minimal. We can still find surface nanobubbles at $T>T_{\mathrm{w}}$ and $\mu<\mu_{\text {sat }}$, but as the wall is completely wet at saturation, such bubbles must detach from the surface at sufficiently small $|\Delta \mu|$.

\section{Interface binding potential}

In this section we introduce a macroscopic description which corrects the spectacular failure of a description in terms of $R_{\mathrm{L}}$ for small bubbles, discussed in the previous section and demonstrated in figure 2 . We further relate this revised macroscopic description with DFT and show how the two can be used in tandem to describe small systems with diffuse interfaces, high degree of non-uniformity and highly non-local interactions in terms of the intuitive macroscopic concepts of sharp interfaces and biding potentials.

It is noteworthy that with DFT we did not need to stipulate the existence of interfaces, instead the film-, drop- and bubble-like density configurations with diffuse fluid-fluid and fluid-wall interfaces were obtained by solving the non-local DFT equation (2.7). In fact the formation of these interfaces is the result of local phase separation, occurring because of the correctly chosen bulk thermodynamic point $(T, \mu)$, near bulk coexistence and wall wetting transition. An alternative mean-field approach to wetting can be developed by assuming the existence of a sharp liquid-gas interface, with a shape $l(x)$ minimising the excess free energy functional $\Omega_{l}[l ; T, \mu]$ of the adsorbate at the given $T$ and $\mu$. Here $l(x)$ is the distance of the liquid-gas interface from the wall. Such local approach can be traced back to the early works of Frumkin and Derjaguin (FD; see, e.g., Henderson (2011) and references therein). Thus, for a system, translationally invariant along the $z$-axis, the interface free energy per unit length can be written in the following general form [see, e.g., Dietrich (1988) and Saam (2009)]:

$$
\Omega_{l}[l ; T, \mu]=\int_{-\infty}^{+\infty}\left[\gamma_{\mathrm{lg}}(T) \sqrt{1+\left(\frac{d l}{d x}\right)^{2}}+W(l(x) ; T, \Delta \mu)\right] d x,
$$

where the first term of the integrand is the free energy per unit area of the liquidgas interface, and the second term is the thermodynamic work of adsorbate formation. Depending on whether the adsorbate is formed of liquid or gas, $W \equiv W_{\text {liq }}$ or $W \equiv W_{\text {gas }}$, respectively, with the corresponding volume and surface contributions:

$$
W_{\text {liq } / \text { gas }}(l(x) ; T, \mu)=\Delta \mu \Delta \rho(T, \mu) l(x)+\gamma_{\mathrm{wl} / \mathrm{g}}(T)+\gamma_{\mathrm{lg}}(T)+g_{\mathrm{liq} / \mathrm{gas}}(l(x) ; T),
$$

where $g_{\text {liq/gas }}(l ; T)$ is the interface binding potential of liquid/gas film, adsorbed at saturation. It accounts for the interatomic interactions between the liquid-gas interface 
and the substrate. For LJ forces, $g_{\text {liq } / \text { gas }}(l ; T)=\mathcal{O}\left(l^{-2}\right)$, as $l \rightarrow \infty$. The EL equation (3.1) becomes

$$
\frac{d^{2} l}{d x^{2}}=\frac{1}{\gamma_{\mathrm{lg}}}\left(1+\left(\frac{d l}{d x}\right)^{2}\right)^{\frac{3}{2}} \dot{W}(l ; T, \mu),
$$

where $\dot{W}(l ; T, \mu)$ denotes the derivative of $W(l ; T, \mu)$ with respect to $l$. Equation (3.3) has been referred to as the augmented Young-Laplace equation by Wu \& Wong (2004), i.e. the standard Young-Laplace equation modified to include the disjoining pressure. Here we shall refer to (3.3) as the FD equation because we establish the connection between the microscopic free energy in (2.1) and the macroscopic DF free energy in (3.1), which in turn leads to (3.3). Without consideration of the free energies, this link cannot be established as disjoining pressure is typically associated with the formation of thin films.

If $W(l ; T, \mu)$ is known, the FD equation (3.3) can be integrated from the absolute minimum of $W(l ; T, \mu)$, until $d l / d x$ becomes constant. Notice that when $\dot{W} \equiv 1$, equation (3.3) defines a circle of curvature $\gamma_{\mathrm{lg}}^{-1}$, and when $\dot{W} \equiv 0$, it defines a straight line. Therefore, at $\mu_{\text {sat }}$ the solution must tend to a flat liquid-gas interface, and below/above $\mu_{\text {sat }}$, it must trace a circular-like shape of a drop/bubble. From equation (3.3), it follows that the curvature of the sharp interface $l(x)$ is given by $\dot{W} / \gamma_{\mathrm{lg}}$, where according to equation (3.2) and the definition of $R_{\mathrm{L}}, \dot{W} \approx \gamma_{\mathrm{lg}} / R_{\mathrm{L}}+\dot{g}$. Thus, $\dot{g} / \gamma_{\mathrm{lg}}$ has a nice interpretation as the curvature correction to the circular shape of the drop/bubble of Laplace radius, induced by the presence of the substrate.

We can compute the work of film formation $W_{\text {liq }}(l ; T, \mu)$ and $W_{\text {gas }}(l ; T, \mu)$ from DFT, using the same density profiles in $\{\rho(x)\}_{T}^{\text {gas }}$ and $\{\rho(x)\}_{T}^{\text {liq }}$ that give rise to the liquidand gas-adsorption isotherms, such as shown in figure 1(a). This requires us to make an ansatz about the density and free energy density profiles of a liquid (or gas) film, of a given height $l$, adsorbed on the wall at the given $T$ and $\mu$. All we know is that such film must be thermodynamically unstable, unless all three, $l, \mu$ and $T$, belong to a (meta)stable branch of an adsorption isotherm. If we ignore the fact that each profile in the isotherm sets is associated with a particular chemical potential and treat $\mu$ as fixed, the sets $\{\rho(x)\}_{T}^{\text {gas }}$ and $\{\rho(x)\}_{T}^{\text {liq }}$ turn out to contain the density profiles of (now unstable) adsorbed films of different widths. Moreover, these density profiles retain the key features of the LJ model of adsorption: a nearly flat plateau at the density of the adsorbed phase, followed by a diffuse liquid-gas interface, consistent with $T$, and an inverse cubic rate of decay to bulk density. Also, the near-wall fluid structure and the interaction of the fluidwall and liquid-gas interfaces at small $l$ are keeping in touch with the underlying DFT functional. Note that since $\mu$ is fixed, the large- $x$ limit of the density profiles so chosen do not coincide with the bulk density at $\mu$. This is not a problem, because $W(l ; T, \mu)$ is the free energy of the adsorbed film, so the bulk contribution has to be removed anyway. At the same time, the rate of decay of the density profile to its asymptote is important.

Thus, given $T, \mu$ and a profile $\rho_{0}(x)$ from $\{\rho(x)\}_{T}^{\text {gas }}$ or $\{\rho(x)\}_{T}^{\text {liq }}$, which solves the EL equation $(2.7)$ at the required $T$ and some $\mu_{0}$, we need to compute the height $l$ of the adsorbed film, and its free energy $W(l ; T, \mu)$. Associated with $\rho_{0}(x)$ are its limit $\rho_{\mathrm{b}}^{0}=\lim _{x \rightarrow \infty} \rho_{0}(x)$, adsorption $\Gamma_{0}=\int_{0}^{\infty}\left(\rho_{0}(x)-\rho_{\mathrm{b}}^{0}\right) d x$, and the grand free energy density $\omega\left(x ; \rho_{0}(x), T, \mu_{0}\right)$, defined in equation $(2.11)$. It is reasonable to set $l=\Gamma_{0} / \Delta \rho(T, \mu)$, because then $l$ is related to the physical bulk density (given by $T$ and $\mu$ ) and correctly recovers $l_{0}\left(T, \mu_{\text {sat }}\right)$ and $l_{0}\left(T, \mu_{0}\right)$, see equation (2.10). Additionally, this ensures that $l$ cannot be "computed" in the region outside the bulk spinodals, where metastable phases (and thus $\Delta \rho$ ) do not exit. The free energy of the adsorbed film can be obtained from $\Omega\left[\rho_{0}(x) ; T, \mu\right]$ in equation $(2.1)$ by removing the contribution of gas on the outside of 

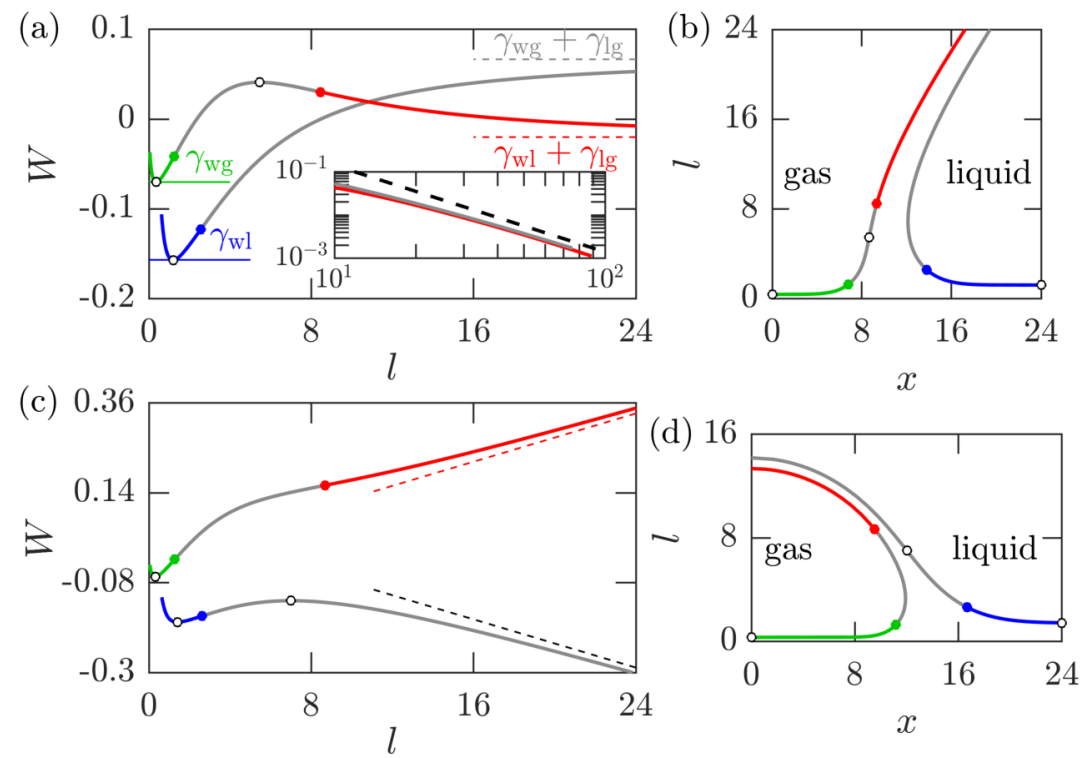

Figure 3. The curve colours and open/filled circles here correspond to those in figure 1(a) at the same $l$. (a) and (c): Work of formation of liquid film, $W_{\text {liq }}(l ; T, \mu)$ (green-grey-red curves), and gas film, $W_{\text {gas }}(l ; T, \mu)$ (blue-grey curves) at $T=0.88\left(\Theta_{\mathrm{Y}}=50.6^{\circ}\right)$. (b) and (d): Liquid-gas interfaces, obtained from equation (3.3) with $W=W_{\text {liq }}$ and $W=W_{\text {gas }}$, and coloured accordingly. In (a) and (b) $\Delta \mu=0$, and in (c) and (d) $\Delta \mu=-0.035$. The spinodals of $l(\mu)$ in figure 1(a) correspond to the inflection points of $W$ (filled circles), and the intersections of $l(\mu)$ with lines $\Delta \mu=0$ and $\Delta \mu=-0.035$ correspond to the extrema of $W$ (open circles). The asymptotes of $W$ are indicated by dashed lines. In (a), the limits and minima of $W\left(l ; T, \mu_{\text {sat }}\right)$ are indicated by text; the inset shows the decay of $W\left(l ; T, \mu_{\text {sat }}\right)$ on a log-log plot, where the black dashed line is a guide to eye at the expected asymptotic decay $W \propto 1 / l^{2}$.

the liquid-gas interface. Because the liquid-gas interface is diffuse, simply limiting the integration interval to $[0, l]$ will be incorrect. The solution is to work instead with the grand free energy density, $\omega\left(x ; \rho_{0}(x), T, \mu_{0}\right)$, defined in equation $(2.11)$. Notice that for large $x, \omega\left(x ; \rho_{0}(x), T, \mu\right)=-P\left(\rho_{\mathrm{b}}^{0}, T\right)+\left(\mu_{0}-\mu\right) \rho_{\mathrm{b}}^{0}+\mathcal{O}\left(1 / x^{3}\right)$ as $x \rightarrow \infty$. Removing this bulk contribution, we get $W(l ; T, \mu)$ by integration:

$$
\begin{array}{r}
W(l ; T, \mu)=\int_{0}^{\infty}\left[\omega\left(x ; \rho_{0}, T, \mu\right)-\left(-P\left(\rho_{\mathrm{b}}^{0}, T\right)+\left(\mu_{0}-\mu\right) \rho_{\mathrm{b}}^{0}\right)\right] d x, \\
l=\int\left(\rho_{0}(x)-\rho_{\mathrm{b}}^{0}\right) / \Delta \rho(T, \mu) .
\end{array}
$$

Repeating this computation for every profile in $\{\rho(x)\}_{T}^{\text {gas }}$ and $\{\rho(x)\}_{T}^{\text {liq }}$, we tabulate the respective work of gas and liquid film formation, $W^{\text {gas }}(l ; T, \mu)$ and $W^{\text {liq }}(l ; T, \mu)$.

In figure 3 we represent the work of film formation at $T=0.85$, and $\Delta \mu=0$ and $\Delta \mu=-0.035$, together with solutions of the FD equation (3.3). The curves $W_{\text {liq }}(l ; T, \mu)$ (green-grey-red) and $W_{\text {gas }}(l ; T, \mu)$ (blue-grey), and the FD interfaces $l_{\text {liq }}(x)$ and $l_{\text {gas }}(x)$, obtained using them, are coloured as the isotherms $l_{\text {liq }}(\mu)$ and $l_{\text {gas }}(\mu)$ in figure $1($ a) at respective $l$. For illustration purposes, we have shifted the curves $l_{\text {gas }}(x)$ along the $x$-axis to facilitate visual comparison with $l_{\text {liq }}(x)$. Notice that the unstable parts of $W(l ; T, \mu)$ curves (grey) are concave, and separated from the convex stable parts (colored) by the 
inflection points (filled circles), which correspond to the spinodals of $l(\mu)$. Local extrema of $W(l ; T, \mu)$ (open circles) correspond to the intersections of $l(\mu)$ with lines $\Delta \mu=0$ and $\Delta \mu=-0.035$. In fact, the qualitative behavior of $W(l ; T, \mu)$ and $l(x)$ is apparent from figure 1(a): intersections of isotherms with vertical lines $\mu=$ const. must correspond to alternating minima and maxima of $W(l ; T, \mu)$.

At saturation [figure 3(a)] the volume contribution to $W(l ; T, \mu)$ vanishes, and $W_{\text {liq }}\left(l ; T, \mu_{\text {sat }}\right)$ and $W_{\text {gas }}\left(l ; T, \mu_{\text {sat }}\right)$ tend to the respective sums of the liquid-gas and fluidwall surface tensions, as $l \rightarrow \infty$. The inverse quadratic rate of decay of $W_{\text {liq }}\left(l ; T, \mu_{\text {sat }}\right)$ can be verified numerically, as shown in the inset of figure 3(a). Subtracting these asymptotes from $W_{\text {liq }}\left(l ; T, \mu_{\text {sat }}\right)$ and $W_{\text {gas }}\left(l ; T, \mu_{\text {sat }}\right)$, would yield the interface binding potential, which turns out to have two branches, associated with liquid and gas adsorption, respectively. The local minima of $W_{\text {liq }}\left(l ; T, \mu_{\text {sat }}\right)$ at $W_{\text {liq }}=\gamma_{\mathrm{wg}}$ and that of $W_{\text {gas }}\left(l ; T, \mu_{\text {sat }}\right)$ at $W_{\text {gas }}=\gamma_{\mathrm{wl}}$ reflect the fact that when saturation is approached from the gas/liquid phase, the wall is covered by a stable layer of liquid/gas, respectively [see green/blue branch in 1(a)]. Since the attractive wall favours liquid, this minimum of $W_{\text {gas }}\left(l ; T, \mu_{\text {sat }}\right)$ must always be global. On the other hand, the first-order wetting transition brings about a competing minimum of $W_{\text {liq }}\left(l ; T, \mu_{\text {sat }}\right)$ at $l \rightarrow \infty$, which becomes global at $T \geqslant T_{\mathrm{w}}$.

The stability of liquid and gas films, expressed by the work of film formation and, equivalently, by the adsorption isotherms, directly translates into the near-substrate shapes of the liquid-gas interfaces $l(x)$, depicted in figure 3(b). At saturation, $l_{\text {liq }}(x)$ must have an inflection point at the same $l$, where $W_{\text {liq }}\left(l ; T, \mu_{\text {sat }}\right)$ has a local maximum. The existence of such local maximum directly follows from the fact that the binding potential must have two minima to allow for first-order wetting transition. On the other hand, $l_{\text {gas }}(x)$ cannot have an inflection point at saturation, because $W_{\text {liq }}\left(l ; T, \mu_{\text {sat }}\right)$ must monotonously approach $\gamma_{\mathrm{wg}}+\gamma_{\mathrm{lg}}$ after reaching its local minimum (there is no coexistence between adsorbed gas films). As mentioned above, the starting point on each $l(x)$ is at the minimum of the respective $W$ (marked by open circle), and the numerical solution is stopped when the change of $d l / d x$ is below machine tolerance. Since at saturation, $\dot{W}(l) \rightarrow 0$ with $l$ as $\mathcal{O}\left(1 / l^{3}\right), l(x)$ must tend to a straight line with an inclination angle $\Theta_{Y}$ to the $x$-axis. Numerically we find that $\Theta_{Y}$, predicted by DFT is reached by $l(x)$ with a remarkable accuracy.

Off of saturation $W(l ; T, \mu)$ can be computed by applying equations (3.4), or from $W\left(l ; T, \mu_{\text {sat }}\right)$, by subtracting the volume term $\Delta \mu \Delta \rho l$ and rescaling $l$ appropriately. In figure 3(c) we applied equations (3.4) and verified numerically that $W(l ; T, \mu)$ has the linear asymptotes $\Delta \mu \Delta \rho l$ (dashed lines). Notice that here the volume term creates a local maximum for $W_{\text {gas }}(l ; T, \mu)$ and eliminates it for $W_{\text {liq }}(l ; T, \mu)$. As a result, the interface line $l_{\text {gas }}(x)$ has an inflection point but $l_{\text {liq }}(x)$ does not [figure $3(\mathrm{~d})$ ]. The interface lines must have "near-circular" shapes when the volume term is nonzero, because if $\dot{W}(l ; T, \mu)$ is a constant, equation (3.3) defines a circle.

The interfaces $l_{\text {liq }}(x)$ and $l_{\text {gas }}(x)$ differ substantially near the wall, reflecting the fact that the wetting transition and thin-thick film coexistence affects $W_{\text {liq }}(l ; T, \mu)$, but not $W_{\text {liq }}(l ; T, \mu)$. Notice also that the effect of the solvation force, locally repelling the liquid from the wall, leads to a higher near-wall part of $l_{\text {gas }}(x)$, than $l_{\text {liq }}(x)$ in figures $3(\mathrm{~b})$ and (c). The DF model captures this effect via the properly computed work of film formation, where the local minimum of $W_{\text {gas }}(l ; T, \mu)$ is reached at a slightly higher $l$, than that of $W_{\text {liq }}(l ; T, \mu)$. It is noteworthy that the part of $l_{\text {liq }}(x)$ between the near-wall region (green) and the outer liquid-gas region (red) is determined by the density profiles $\rho(x)$ from the saddle manifold of $\Omega[\rho(x)]$. Thus, the mean-field DFT functional allows us to connect 

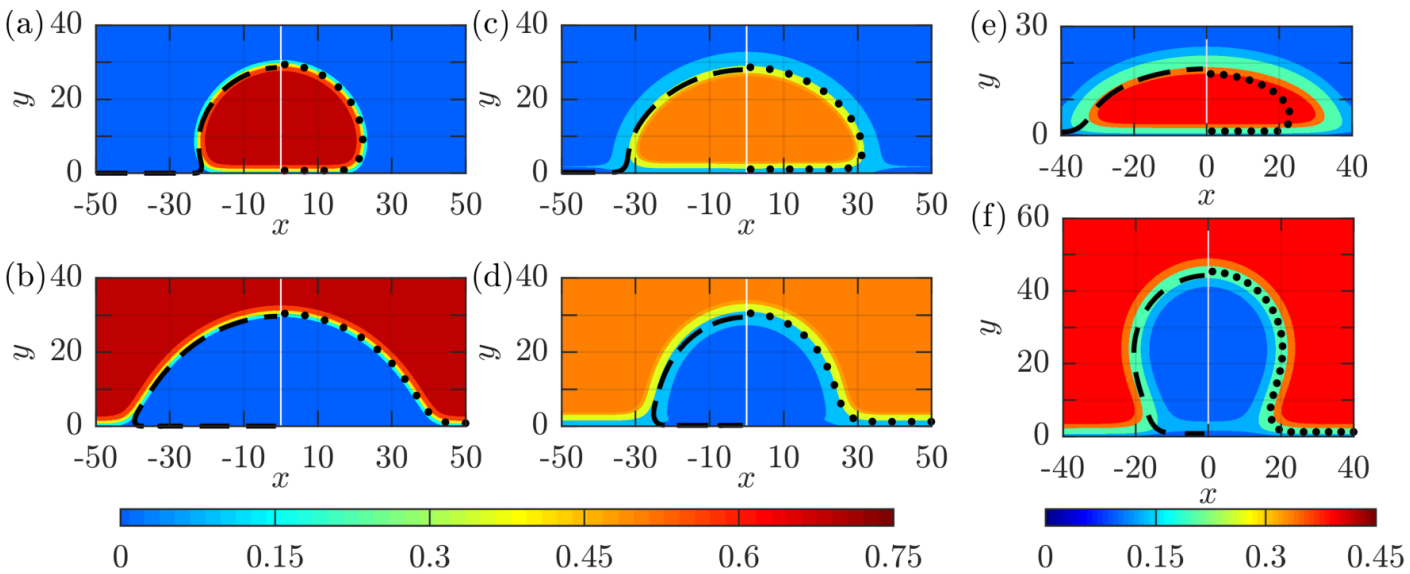

$$
\begin{array}{llllll}
0 & 0.15 & 0.3 & 0.45 & 0.6 & 0.75
\end{array}
$$

0

$0.15-0.3$

0.45

Figure 4. Comparison between DFT (coloured contour plots) and DF model (black curves). In each plot, dashed and dotted black curves to the left and right of the white vertical line at $x=0$ correspond to $l_{\text {liq }}(x)$ and $l_{\text {gas }}(x)$, respectively. In (a)-(d) $T<T_{\mathrm{w}}$; the drops and bubbles have the same average height $30 \sigma$. In (e)-(f) $T>T_{\mathrm{w}}$, and we see a nucleation cluster of the flat prewetting film, and a detaching bubble. Thermodynamic points: [(a) and (b)]: $T=0.65\left(\Theta_{\mathrm{Y}}=101^{\circ}\right)$, $\Delta \mu=-2.4 \times 10^{-2}$ and $3.8 \times 10^{-2}$. [(c) and $\left.(\mathrm{d})\right]: T=0.85\left(\Theta_{\mathrm{Y}}=65^{\circ}\right), \Delta \mu=-1.8 \times 10^{-2}$ and $0.9 \times 10^{-2} \cdot[(\mathrm{d})$ and $(\mathrm{f})]: T=0.93\left(\Theta_{\mathrm{Y}}=0^{\circ}, \Delta \mu_{\mathrm{pw}}=-1.4 \times 10^{-3}\right), \Delta \mu=-8.5 \times 10^{-4}$ and $-1.0 \times 10^{-2}$.

these metastable states in a non-ambiguous way. In the case of $l_{\text {gas }}(x)$, the density profiles from the saddle manifold of $\Omega[\rho(x)]$ determine most of the liquid-gas interface.

In figure 4 we represent a direct comparison between the DFT density profiles and DF interface lines $l_{\text {liq }}(x)$ (dashed) and $l_{\text {gas }}(x)$ (dotted), obtained at the same $T$ and $\mu$. To superimpose the DF interfaces over $\rho(x, y)$, we aligned the tops of $l_{\text {liq }}(x)$ and $l_{\text {gas }}(x)$ (where $d l / d x$ vanish) with $x=0$. We also reflected some $l(x)$ with respect to the $y$-axis, so that $l_{\text {liq }}(x)$ and $l_{\text {gas }}(x)$ appear in $x \leqslant 0$ and $x \geqslant 0$ half-planes of each plot, respectively. In figures 4 (a)-(c) we show nanodrops and -bubbles of the same height $30 \sigma$ at two different temperatures below $T_{\mathrm{w}}$. Overall, the local DF model captures well the heights of the drops and bubbles, and the changes of the interface curvature at small scales. As can be seen, $\rho(x, y)$ possesses level sets which closely resemble both $\rho_{\text {liq }}$ and $\rho_{\text {gas }}$. Interestingly, no part of $l_{\text {liq }}(x)$ can be found between the wall and liquid, just as no part of $l_{\text {gas }}(x)$ is found between the wall and gas. This is a limitation of the local functional $\Omega_{l}[l]$, which does not allow $l(x)$ to bifurcate. Another limitation of the DF model, not apparent from figure 4, was already mentioned above: our treatment of repulsions in equation (2.2) cannot capture the near-wall layering of the fluid density associated with the excluded volume intermolecular correlations. This is because our DFT functional was chosen specifically to capture the liquid-gas interface, without the unnecessary complications of the finer nearwall details, which are irrelevant for the present study. Investigating the low-temperature regime, where the near-wall layering is important certainly presents interesting questions for further study, beyond the scope of the present work.

It is very important to understand that a local model, such as DF cannot in principle be expected to replace a statistical-mechanical method, such as DFT. An excellent example illustrating the tight limits of applicability of local models is provided by the continuous pre-wetting transition (Yatsyshin et al. 2016). Since we have a microscopic drop on a substrate, it must nucleate a thin liquid film at $\mu_{\mathrm{pw}}$ and $T>T_{\mathrm{w}}$ (see figures 1 (a) and (b)). While DFT captures the onset of such pre-wetting without any problem, the DF model breaks down, because it does not include the interactions between different points 
on the interface. In figure 4(e) we depict a nucleation cluster of the flat pre-wetting film above $T_{\mathrm{w}}$. The round edges of the adsorbed liquid structure are deceptive. Fitting a circle to the liquid-gas interface as in figure 2 , we get $R^{*}=49 \sigma$. At the same time, the Laplace radius $R_{\mathrm{L}}=225 \sigma$, testifying to the fact that what we see is indeed a forming flat film. The work $W_{\text {gas }}(l ; T, \mu)$ does not include the bi-stability of wetting liquid films, and as a result $l_{\text {gas }}(x)$ fails to capture the lateral extent of the film. This failure of $l_{\text {gas }}(x)$ near prewetting transition is systematic in our study and manifests the highest disagreement between the DF and DFT models.

Rather surprisingly, we find a remarkable agreement between the local DF and nonlocal DFT in the case of a nearly detached nanobubble, shown in figure 4(e) [since $\Theta_{\mathrm{Y}}=0$, the surface bubbles will detach as $\Delta \mu \rightarrow 0]$. The DF interface lines here are S-shaped (although still single-valued if treated as functions of $y$ ), as are the level sets of $\rho(x, y)$. This is curious, because the local free energy $\Omega_{l}$ does not account for the self-interaction in the liquid-gas interface, and the agreement between DF model and DFT should have broken down. It follows that such S-shaped profiles are the consequence of the change of sign of $\dot{W}$ in equation (3.3), and do not necessarily require including curvature and self-interaction effects into the interfacial free energy $\Omega_{l}$.

\section{Conclusions}

We have presented a statistical-mechanical study of thermodynamics and interface shapes of surface nanodrops and nanobubbles. The starting point is a DFT framework based on modelling the fluid-fluid and fluid-substrate interactions at the atomic level. For this purpose we adopted the LJ potential (1.1). At the same time, DFT accounts for the non-locality of interatomic interactions, as manifested by the integral EL equation (2.7) for the fluid density profiles. Because the thermodynamics of liquid-gas coexistence is fully incorporated in DFT, we did not need to assume the existence of fluid-fluid or fluid-wall interfaces as is often the case with macroscopic models of wetting. Given the right thermodynamic point $(T, \mu)$ near both bulk coexistence and wall wetting transition, diffuse interfaces appear naturally, as a result of local phase separation. We found that the regions in the thermodynamic space of temperature and chemical potential where nanodrops and nanobubbles exist are demarcated by the spinodals of planar wetting films. Remarkably, this implies that surface nanobubbles may be more amenable to experimental study than nanodrops, precisely because they occupy a bigger portion of the phase plane. Additionally the thin film spinodals can be used to compute the minimal radii of surface drops and bubbles.

Although valuable insight about the contact line region can be gained from microscopic DFT computations, large drops and bubbles are computationally expensive with DFT. On the other hand, DFT computations clearly show that small bubbles are not spherical caps, and descriptions relying on macroscopic concepts, such as sharp interfaces, contact angles and binding potential, need to appreciate this fact. We provided an example in terms of a simple DF model, which manages to capture the intricate and subtle aspects of non-sphericity of the nanodrop/bubble shapes by accounting for the binding potential. Furthermore, we demonstrated how the binding potential can be related to the much more consistent statistical mechanical treatment of nanodrops/bubbles with DFT. The method of computing the interface binding potential by renormalising the DFT isotherms is certainly promising in a broader context, e.g., as parameter passing between microscopic statistical mechanical approaches and continuum mechanical treatments, such as long wavelength approximation to Navier-Stokes equations. Our results for binding potential further highlight the important role played by planar wetting in the physics of surface 
drops and bubbles. For example, from figure 1(a) it follows that above the thin film spinodal, $W_{\text {liq }}(l ; T, \mu)$ will not have a local minimum. Thus, although the Laplace radii are non-zero above the thin film spinodal, the wall wetting mechanism does not allow the drops to form.

Very interestingly, applying the binding potential to compute the contact line of macroscopic drops we find two possible behaviours, depending on whether liquid-gas coexistence is reached from the liquid or gas sides of phase diagram. Usually $g(l ; T)$ is being associated with a liquid film. However, from figure $3(\mathrm{a})$ if follows that $g(l ; T)$ must be multivalued, consisting of two (disconnected) branches, corresponding to adsorption of liquid and gas. Of-course, in a purely equilibrium setting only one of the configurations $l_{\text {liq }}(x)$ are to be observed, because $l_{\text {gas }}(x)$ will have a higher free energy, due to the wall favouring liquid. Nevertheless, in a dynamic setting, such as droplet spreading (Engelnkemper \& Thiele 2019; Kirkinis \& Davis 2017), the metastable states $l_{\text {gas }}(x)$ may be manifested as contact line hystersis. Additionally, introducing a sizable impurity into the substrate, may make $l_{\text {gas }}(x)$ the global minimiser of the interfacial free energy.

In our computations, the bulk phase is metastable. This should be viewed as a necessary restriction of the model and may be revisited by investigating pinned drops and bubbles, which will be thermodynamically stable. Another restriction of the present approach is inherent in its mean-field nature, in that interface fluctuations are not accounted for. Nevertheless, far from criticality, mean-field is known to provide reliable predictions for most practically interesting cases.

There is much experimental and theoretical interest in understanding the behaviour of apparent contact angles of small drops/bubbles (Qian et al. 2019). Our computations demonstrate that the apparent contact angles, such as those shown in figure 2, are highly sensitive to the details of the definition of a sharp interface, as well as the fitting procedure. One needs to be very careful when analysing contact angles using simulation or experimental data for small drops/bubbles, and performing relevant statistical sensitivity analysis (Che \& Theodorakis 2017). The agreement we get between DFT and the sharp interface model in equation (3.3) suggests that rather than fitting spherical caps to nanodrops/bubbles, one should probably attempt to fit a model accounting for disjoining pressure effects, such as (3.3). This can be done using modern data-analysis techniques.

We note that DFT models for repulsions of higher sophistication than the local density approximation in equation (2.2) will reveal all the effects we describe in the present work, because these effects are caused by the molecular attractions and are captured by the random phase approximation for the attractive free energy in equation (2.4). Nevertheless, more sophisticated repulsive DFT functionals, such as FMT, will facilitate the study of additional effects, associated with excluded volume molecular interactions and correlations, and the resulting short-ranged near-wall layering of the density profiles at distances of 1-5 molecular diameters from the wall. One of our key findings is that the pre-wetting transition enables stabilisation of drops of liquid on vanishingly small impurities on the substrate. Certainly this prompts the existence of a similar relation for low-temperature layering transitions. Investigating these effects is certainly of interest in future work and requires using a repulsive functional with a fully non-local treatment of repulsions. In addition to the effects shown here for molecular attractions, there may appear new minima of the binding potential at low temperatures, associated with the layering transitions and the higher role of molecular repulsions. This will certainly enrich the picture of adsorption presented and could potentially form a very promising direction for future work. 


\section{Acknowledgements}

We are indebted to Professor Andrew O. Parry and former group member Dr. Miguel Durán-Olivencia for numerous stimulating discussions. We acknowledge financial support from the European Research Council via Advanced Grant No. 247031 and from EPSRC via Grant Nos. EP/L020564, EP/L027186/1 and EP/K503733 (EPSRC-Imperial College Pathways to Impact-Impact Acceleration Award).

\section{REFERENCES}

Bonn, D. \& Ross, D. 2001 Wetting Transitions. Rep. Prog. Phys. 64, 1085.

Dietrich, S. 1988 Wetting Phenomena. In Phase Transitions and Critical Phenomena (ed. C. Domb \& J. L. Lebowitz), , vol. 12, p. 2. Academic Press.

Evans, R. 1990 Microscopic Theories of Simple Fluids and Their Interfaces. In Les Houches 1988. Liquids at Interfaces. (ed. J. Charvolin, J. F. Joanny \& J. Zinn-Justin), p. 1. NorthHolland.

Hauge, E. H. 1992 Macroscopic Theory of Wetting in a Wedge. Phys. Rev. A 46, 4994.

Henderson, D. 2011 Disjoining pressure of planar adsorbed films. Eur. Phys. J. Special Topics $197,115$.

Hughes, A.P., Thiele, U. \& Archer, A.J. 2017 Influence of the fluid structure on the binding potential: Comparing liquid drop profiles from density functional theory with results from mesoscopic theory. J. Chem. Phys. 146 (6), 064705.

Theodorakis, P. E. \& Che, Z. 2019 Surface nanobubbles: Theory, simulation, and experiment. A review. Adv. Colloid Interfac. 272, 101995.

Qian, J., Arends, G. F. \& Zhang, X. 2019 Surface Nanodroplets: Formation, Dissolution, and Applications. Langmuir 35, 12583.

Lohse, D. \& Zhang, X. 2015 Surface Nanobubbles and Nanodroplets. Rev. Mod. Phys. 87, 981.

Lutsko, J. F. 2010 Recent Developments in Classical Density Functional Theory. In Adv. Chem. Phys., p. 1. John Wiley \& Sons.

MaCDowelL, L. G. 2011 Computer simulation of interface potentials: Towards a first principle description of complex interfaces? The European Physical Journal Special Topics 197 (1), 131-145.

MacDowell, Luis G., Benet, Jorge, Katcho, Nebil A. \& Palanco, Jose M.G. 2014 Disjoining pressure and the film-height-dependent surface tension of thin liquid films: New insight from capillary wave fluctuations. Adv. Colloid Interfac. 206, 150-171.

Nold, A., Sibley, D.N., Goddard, B.D. \& Kalliadasis, S. 2014 Fluid structure in the immediate vicinity of an equilibrium three-phase contact line and assessment of disjoining pressure models using density functional theory. Phys. Fluids 26 (7), 072001.

Pereira, A. \& Kalliadasis, S. 2012 Equilibrium Gas-Liquid-Solid Contact Angle From Density-Functional Theory. J. Fluid Mech. 692, 53-77.

Rascón, C. \& PARry, A. O. 2000 Geometry-Dominated Fluid Adsorption on Sculpted Solid Substrates. Nature 407, 986.

Roth, R. 2010 Fundamental Measure Theory for Hard-Sphere Mixtures: A Review. J. Phys.: Condens. Matter 22, 063102.

Rowlinson, J. S. \& Widom, B. 1982 Molecular Theory of Capillarity. Dover, New-York.

SaAm, W. F. 2009 Wetting, Capillary Condensation and More. J. Low Temp. Phys. 157, 77.

Sullivan, D. E. \& Telo Da Gama, M. M. 1986 Wetting Transitions and Multilayer Adsorption at Fluid Interfaces. In Fluid Interfacial Phenomena (ed. C. A. Croxton), p. 45. Wiley, New York.

Svetovoy, V. B., Devic, I., Snoeijer, J. H. \& Lohse, D. 2016 Effect of Disjoining Pressure on Surface Nanobubbles. Langmuir 32, 11188.

Wu, J. \& Li, Z. 2007 Density-Functional Theory for Complex Fluids. Annu. Rev. Phys. Chem. $\mathbf{5 8}, 85$.

Wu, Q. \& Wong, H. 2004 A slope-dependent disjoining pressure for non-zero contact angles. J. Fluid Mech. 506, 157-185. 
Yatsyshin, P. \& Kalliadasis, S. 2016 Mean-field Phenomenology of Wetting in Nanogrooves. Mol. Phys. 114, 2688.

Yatsyshin, P., Parry, A. O. \& Kalliadasis, S. 2016 Complete Prewetting. J. Phys.: Condens. Matter 28, 275001.

Yatsyshin, P., Parry, A. O., Rascón, C. \& Kalliadasis, S. 2017 Classical Density Functional Study of Wetting Transitions on Nanopatterned Surfaces. J. Phys.: Condens. Matter 29, 094001.

Yatsyshin, P., Savva, N. \& Kalliadasis, S. 2015 Density Functional Study of Condensation in Capped Capillaries. J. Phys.: Condens. Matter 27, 275104.

Yin, H., Sibley, D.N. \& Archer, A.J. 2019 Binding potentials for vapour nanobubbles on surfaces using density functional theory. J. Phys.: Condens. Matter 31 (31), 315102.

Engelnkemper, S. \& Thiele, U. 2019 The collective behaviour of ensembles of condensing liquid drops on heterogeneous inclined substrates Europhys. Lett. 127 , 54002.

KIRKINIS, E. \& DAVIS, S.H. 2013 Hydrodynamic theory of liquid slippage on a solid substrate near a moving contact line Phys. Rev. Lett. 110 , 234503.

Theodorakis, P. E. \& Che, Z. 2017 Formation, dissolution and properties of surface nanobubbles J. Colloid Interface Sci. 487, 123. 